ARTÍCULOS ORIGINALES Rev Chil Salud Pública 2015; Vol 19 (2): 140-153

\section{ECOS DE MARX Y MALTHUS: EL CAMINO ROCOSO DESDE EL CONTROL DE LA POBLACIÓN HACIA LOS DERECHOS REPRODUCTIVOS}

\author{
Echos of Marx and Malthus: the ROCKY ROAD from \\ POPULATION CONTROL TO REPRODUCTIVE RIGHTS
}

\section{RESUMEN}

En este estudio enlazo debates sobre la regulación de la fertilidad bumana y el control de la población con las consideraciones sobre los derechos reproductivos de la mujer. Primero, una breve discusión de antiguas rivalidades entre Thomas Robert Malthus y Karl Marx introduce el carácter profundamente político de las consideraciones sobre reproducción bumana. A continuación me traslado al siglo $X X$ y muestro que abora reencontramos a Marx y a Malthus vestidos en ropa nueva por rivales politicos que aplicaron antiguas rivalidades a nuevos conflictos politicos. Me concentro en el periodo posterior a la Segunda Guerra Mundial, cuando los nuevos debates sobre población y derechos reproductivos estaban acompañados por los nuevos paradigmas globales de los derechos bumanos -y argumento que la práctica de los derechos estaba comprometida por los enfrentamientos entre politicos de derecha e izquierda-presentados durante la Guerra Fría. Apoyándome en historias de médicos y planificadores poblacionales activos en Europa y las Américas, me concentro en el estudio del caso de la planificación familiar $y$ los derechos reproductivos en Chile para mostrar los efectos dañinos que los enfrentamientos de la Guerra Fría -las dicotomías politicas de derecha e izquierda-tuvieron en las políticas de salud y de derechos reproductivos.

Palabras clave: Neo-Malthusianismo, planificación familiar, Guerra Fría, derechos reproductivos.

\begin{abstract}
In this study, I link key debates about the regulation of buman fertility and population control to considerations about women's reproductive rights. First, a brief discussion of old rivalries between Thomas Robert Malthus and Karl Marx introduces the deeply political nature of considerations about buman reproduction. Next, I move from the 18th and 19th centuries to the 20th century, and show that we find Marx and Malthus dressed in new clothes by political rivals who applied old competitions to new political conflicts.I focus on the post WW II period, when new debates about population and buman reproduction were accompanied by new global paradigms of human rights -and argue that a twentieth century practice of rights was compromised by competitions between politics of left and right-set in the Cold War.
\end{abstract}

Recibido 24 de marzo 2015, aceptado 31 de marzo 2015 
Drawing on histories of medical doctors and population planners who were active in Europe and the Americas, I focus on the case study of family planning and reproductive rights in Chile to show the ill-effects that Cold War competitions -the political dichotomies of left and right-had on the politics of health and reproductive rights.

Keywords: Neo-Malthusianism, family planning, Cold War, reproductive rights.

\section{INTRODUCCIÓN: LOS USOS POLÍTICOS DE MALTHUS EN UNA ERA DE CAMBIO REVOLUCIONARIO}

A veces nos olvidamos cuán política (y subjetiva) puede ser la supuestamente racional investigación científica, y la investigación poblacional es un ejemplo de ello. Académicos como David Harvey y Armand Mattelart nos recuerdan que los estudios científicos sobre la población humana se presentan a menudo con puntos débiles, que los métodos científicos utilizados para determinar la proporción entre la población y los recursos son altamente problemáticos, y, lo más importante, que la ciencia no es ni apolítica ni éticamente neutral. ${ }^{1,2}$ El texto Ensayo sobre el Principio de la Población del reverendo inglés Thomas Malthus de 1789, por ejemplo, contradijo las esperanzas de una mayor igualdad entre los seres humanos y a las expectativas de progreso social recién traídas por la Revolución francesa. Malthus advirtió sobre lo que él percibió como las consecuencias peligrosas de un crecimiento descontrolado de la población, pues el aumento exponencial de la población humana superaría a los recursos y a la producción de alimentos, llevando al agotamiento. A medida que los pobres de Inglaterra -al igual que en el resto de Europa- superaban en número a los ricos, el estudio de Malthus expresaba que no solo las poblaciones humanas se dirigían a un futuro problemático a menos que aprendieran a limitar su propia reproducción, sino también que el aumento del número de pobres presagiaba consecuencias horribles e incontrolables si las élites se quedaban de brazos cruzados: "El poder de la población es tan superior al poder de la tierra para producir la subsistencia del hombre que la muerte prematura, de alguna forma u otra, debe visitar a la raza humana".

Teniendo en cuenta los temores de las élites europeas que se sentían amedrentadas por la pérdida de poder y privilegio en las postrimerías de la Revolución francesa, no es de extrañar que el texto de Malthus se convirtiese en uno de los documentos más frecuentemente leídos de su tiempo. Su interpretación puede haber representado el arma antirrevolucionaria más útil y la herramienta intelectual más efectiva que justificaba el control de la élite sobre las empobrecidas masas. En pocas palabras, los líderes políticos preocupados y los ciudadanos adinerados podían argumentar que la procreación excesiva, más que las instituciones políticas y económicas que reproducían las desigualdades sociales, causaron la miseria de los pobres y los problemas sociales que plagaban su nación. En este contexto, el sociólogo John Bellamy Foster pudo haber estado en lo cierto al afirmar que "ninguna otra obra fue más odiada por la clase obrera inglesa, ni tan fuertemente criticada por Marx y Engels". ${ }^{4}$ La teoría de Malthus podría ser utilizada como una "justificación” por las clases dirigentes que trataron de fundamentar nuevas medidas para controlar a la población.

Karl Marx y Friedrich Engels consideraban a Malthus como un "adulador de las clases dirigentes”, condenando duramente su predicción de una crisis en la subsistencia humana si el crecimiento de la población no se limitaba. Malthus afirmaba que un incremento exponencial en la población iba a condenar a la hambruna y a la muerte a miles de seres humanos, una profecía que Marx y Engels denunciaban como una "blasfemia repulsiva en contra del hombre y de la naturaleza". ${ }^{5}$ Ellos 
advirtieron que "la consecuencia de esta teoría es que desde que son precisamente los pobres quienes constituyen el excedente de esta población, nada debiera hacerse por ellos". Marx creía que el obscuro dilema de Malthus de un excedente poblacional hambriento solo podría surgir bajo el capitalismo. Una sociedad socialista, mientras tanto, respaldaría cualquier número de personas si solo el sobrante del producto del trabajo, apropiado por los dueños de los medios de producción en las sociedades capitalistas, fuera retornado a los trabajadores, eliminando de esta manera la causa de la pobreza. Ciudadanos productivos, trabajadores, según Marx, siempre serán capaces de producir para ellos y la sociedad. ${ }^{6}$ En resumen: podemos confirmar algunos de los usos políticos de las posturas opuestas sobre la relación entre el tamaño de la población humana y los recursos. Nopretendo sacar conclusiones sobre la interpretación más precisa de Marx y Malthus como teóricos sociales.Aquíme preocupan los usos políticos de sus obras -y las realidades de los derechos reproductivos de las mujeres- que se desarrollaron en el contexto de las posiciones políticas sobre la supuesta necesidad del control del crecimiento poblacional en el mundo después de la Segunda Guerra Mundial.

\section{PLANIFICACIÓN, DERECHOS E HISTORIA}

En el proceso de explorar las realidades cambiantes de la regulación de la fertilidad y los derechos reproductivos, me inspiro en el historiador Diego Armus y su propuesta de escribir una nueva historia de la medicina y de la salud pública.? Según Armus, la historia tradicional de la medicina se centra a menudo en alabar a los grandes médicos y trata de reconstruir lo que es, supuestamente, el "progreso inevitable" de la medicina moderna. Armus nos hace recordar que a veces tenemos que cuestionar la idea del progreso lineal, y ver que el trabajo de los médicos se desarrolla en condiciones inciertas. Debemos tomar en cuenta no solo las incertidumbres políticas, las contradicciones y las circunstancias subjetivas que conforman también la práctica médica, sino además aspectos de po- der. Michel Foucault ha abordado la relación entre el poder y el conocimiento -que también afecta la relación entre los médicos y sus pacientes-. Tenemos que considerar que la modernidad médica significó también nuevos procedimientos invasivos, nuevos modos de controlar el cuerpo humano.

En los años sesenta, nuevas tecnologías anticonceptivas, principalmente la píldora, transformaron el debate sobre la regulación de la reproducción humana en el mundo entero. En realidad, la nueva tecnología tuvo implicaciones revolucionarias. Algunos argumentaban que los avances en tecnologías anticonceptivas podrían proporcionar bases materiales para la igualdad de género, dado que el control sobre el embarazo y maternidad podría expandir las opciones de vida de las mujeres. Otros enfatizaban las nuevas opciones para manejar o controlar el tamaño de la población. Efectivamente, la tecnología anticonceptiva proporcionó una base importante para la igualdad de género -pero el acceso a la tecnología, o la existencia de la píldora, no garantizó automáticamente un aumento en el poder de las mujeres y tampoco garantizó derechos reproductivos-. La historia de la planificación familiar es también una historia de los esfuerzos por controlar los cuerpos de las mujeres.

Demuestro que en la historia de la planificación familiar en Chile, los cambios en los paradigmas globales -así como acontecimientos nacionales y las acciones de médicos chilenos- han sido poderosos determinantes para las opciones de las mujeres. Por lo tanto necesitamos utilizar un lente global para examinar la manera en la que los intereses de los Estados y las instituciones se entrelazaron para obtener conclusiones sobre las opciones que las mujeres tuvieron con respeto a la maternidad voluntaria. Médicos que se acercaron al tema de la planificación familiar (aunque desde múltiples perspectivas), nos ayudan a ver las intrincadas conexiones en estos niveles. El doctor Jaime Zipper, por ejemplo, colaboraba con médicos-científicos en contextos internacionales, y ganó prestigio con la promoción de un dispositivo intrauterino (DIU) de su propio diseño. Benjamín Viel, quien fue, al igual 
que Zipper, un médico chileno con presencia internacional, fue figura clave en la fundación tanto del sistema chileno de Salud Pública como de la primera oficina de planificación familiar en Santiago. Viel fue educado en Chile, y también en la Universidad de Harvard y la Universidad John Hopkins, en los Estados Unidos. Consiguió el apoyo económico de la Fundación Rockefeller y otras fuentes norteamericanas para los programas de planificación familiar en Chile. Inspirada por la reflexión del sociólogo Jorge Gaete, quien busca el sentido de las indagaciones sobre los grandes médicos del pasado en Chile, trato de entender no solo las vidas de los médicos chilenos, sino también su mundo -y los conflictos políticos globales- que influyeron en las existencias de los maestros de la medicina. ${ }^{8}$

Aunque el enfoque de este análisis es la historia chilena, es necesario reconocer el impacto de las tensiones de la Guerra Fría y tomar seriamente las aseveraciones de historiadores como Matthew Connelly, quien hace hincapié en que la Guerra Fría fue una lucha para controlar poblaciones, no solamente territorios, con dos potencias vigilando sus fronteras biopolíticas. ${ }^{9}$ En las Américas y en Chile, el número de habitantes de la nación siempre ha sido un tema políticamente delicado, y aún más en el período posterior a la Segunda Guerra Mundial. Claramente, las disputas políticas globales han influido en las opciones de los individuos en niveles tan íntimos como los embarazos y la regulación de la fertilidad. Por ello argumento que cualquier historia de los derechos reproductivos necesita examinar la manera en la que las consideraciones geopolíticas, y las políticas oficiales de población, reforzaron o debilitaron las elecciones que pudieron tomar las mujeres y las parejas sobre embarazos.

\section{CONTEXTOS HISTÓRICOS DE LA REGULACIÓN DE LA FERTILIDAD Y LAS RAÍCES DE LOS PROGRAMAS DE PLANIFICACIÓN FAMILIAR}

La historia oficial institucional de la planificación familiar en Chile se inició en la década de los años sesenta -inspirada por las inicia- tivas de los médicos chilenos para reducir la mortalidad materna causada por abortos inducidos-. Desde principios del siglo XIX, en respuesta a Malthus, los líderes políticos y los reformadores frecuentemente ligaban la planificación familiar a las preocupaciones sobre el tamaño de la población y a los temores de la sobrepoblación. No podemos documentar cuántos individuos y parejas se sintieron obligados a limitar los embarazos debido al terrible dilema malthusiano, pero sabemos que la tarea de prevenir los embarazos era desalentadora y poco fiable en el mejor de los casos. Las nuevas tecnologías médicas ofrecieron posibilidades de una anticoncepción eficaz y fiable solo en el siglo XX. También tenemos evidencia de que muchas mujeres, en todo el mundo, recurrieron a abortos inducidos como uno de los pocos métodos a su alcance para limitar los nacimientos.

Antes de mediados del siglo XX, las parejas chilenas, al igual que aquéllas en otras partes del mundo, utilizaron métodos tales como condones, coitus interruptus, abstinencia sexual y abortos autoinducidos para prevenir y terminar embarazos. Los médicos reconocieron (y documentaron, por primera vez) el problema de abortos inducidos inseguros en la década de los treinta del siglo pasado; en la misma década, los miembros del recién fundado Movimiento Pro-Emancipación de la Mujer Chilena (MEMCh) comenzaron a abordar la necesidad de las mujeres de tener acceso a métodos anticonceptivos y abortos legales. Mientras que los y las activistas del MEMCh impulsaron exitosamente algunos cambios en la vida de las mujeres, incluyendo el sufragio femenino, sus campañas por la liberación de las mujeres de la "maternidad obligada" no llevaron a nuevas políticas en materia de regulación de la fertilidad. Durante décadas, la ilegalidad del aborto fue un obstáculo para cualquier debate público sobre los problemas de las mujeres que habían tenido abortos. El aborto inducido fue declarado delito en el Código Penal chileno de 1874, y aun cuando el aborto terapéutico fue legalizado en Chile en 1931, pocas mujeres tenían los recursos o las conexiones para buscar apoyo oficial legal y médico. 
A continuación, los códigos moralesculturales chilenos que definieron los temas "admisibles" para el debate público impusieron un silencio sobre la sexualidad y la procreación, e impidieron iniciativas "públicas" en respuesta a los problemas "privados" de las mujeres que murieron como resultado de abortos realizados en malas condiciones. Bonnie Shepard argumenta convincentemente que la salud femenina fue también dañada por lo que ella llama un "sistema de doble discurso" de Chile que limitó el acceso de las mujeres a la información y asesoramiento médico..$^{10}$ De hecho, el silencio público sobre el tema del aborto solo fue interrumpido por medio de eventos excepcionales tales como la Convención Médica de 1936 en Valparaíso. Andrea del Campo documenta que en esa reunión algunos médicos de salud pública defendieron fuertemente no solo la legalización del aborto, sino también la distribución de tecnologías anticonceptivas con el objetivo de poner fin a los abortos criminales clandestinos y disminuir la mortalidad materna. Usaron justificaciones que revelaron posturas tanto neomalthusianas como eugenésicas: concluyeron que el tener menos niños, de hecho, no solo aliviaría las vidas de las madres y las familias de la clase trabajadora, sino que también tendría consecuencias positivas para el desarrollo de la población chilena. Algunas de sus propuestas provocaron indignación -y demostraron el profundo conservadurismo social que silenció contemplaciones públicas de la maternidad voluntaria-. Muchos médicos -incluso grupos de profesionales de la salud como las matronas- juzgaron todas las deliberaciones sobre aborto como inmorales, y como ataques directos en contra de la familia y la nación. ${ }^{11}$ Los médicos encontraron nuevos espacios para hacer frente a sus preocupaciones sobre la mortalidad materna solo después de la Segunda Guerra Mundial, cuando los nuevos servicios de salud llevaron a profesionales de la medicina social a los hogares de sus pacientes, y cuando los temores del crecimiento de la población de la posguerra, así como los enfrentamientos de la Guerra Fría añadieron autoridad a los argumentos neomalthusianos.
En la década de los cincuenta del siglo $\mathrm{XX}$, los médicos se dispusieron a reexaminar los problemas de salud en las comunidades urbanas $-\mathrm{y}$ presentaron nuevas estadísticas sobre el aborto y la mortalidad materna-. Descubrieron no solo que las mujeres desesperadamente querían servicios anticonceptivos para limitar sus embarazos; también documentaron que esos mismos servicios anticonceptivos podrían ayudar a resolver la crisis del aborto inducido y la mortalidad materna. Mostraron la correlación entre el número de embarazos y el número de abortos en mujeres: aquellas pacientes que habían tenido muchos embarazos sucesivos -a veces veinte consecutivos-, también tenían historias de múltiples abortos. ${ }^{12}$ Epidemiólogos, como Hernán Romero, Tegualda Monreal, Rolando Armijo, y Mariano Requena, se comprometieron en proyectos de investigación de campo que incluyeron entrevistas en los hogares $\mathrm{y}$ visitas a comunidades locales, indagando sobre las causas de los abortos y las medidas necesarias para evitarlos. Las mujeres afirmaron que las presiones económicas, la ansiedad sobre las condenas morales de los niños nacidos fuera del matrimonio, el abuso sexual, así como su incapacidad para limitar los embarazos, fueron las razones principales para abortos clandestinos que a menudo tuvieron lugar en condiciones insalubres. Los datos también confirmaron que la pobreza por sí sola no explica las altas tasas de aborto; sectores de ingresos medios y altos se encontraban entre el grupo de alto riesgo de las mujeres que buscaron abortos para limitar el tamaño de la familia. Testimonios de mujeres, así como el gran número de abortos inducidos y muertes maternas en ese momento, fueron muy reveladores sobre la necesidad de éstas de control natal. Ellas interrumpieron alrededor de un tercio de todos los embarazos. ${ }^{13,14}$

El año 1961, en cuatro mil entrevistas a mujeres entre 20 y 49 años de edad se demostró que una de cada cuatro había tenido entre uno y 35 abortos inducidos. ${ }^{15}$ Los médicos presentaron estadísticas que mostraban que cerca de un tercio de las mujeres que abortaban requerían de atención médica inmediata. Las complicaciones por los abortos inducidos 
representaban el 8,1\% de las admisiones de todos los hospitales a lo largo del país -y los casos admitidos en los hospitales por complicaciones derivadas de un aborto representaban el $35 \%$ de las cirugías en los servicios de obstetricia y el $26,7 \%$ de la sangre usada en todos los servicios de emergencia- ${ }^{16}$ Los médicos también demostraron que había una correlación directa entre el acceso de las mujeres a la planificación familiar y la subsecuente disminución de abortos inducidos, y de la mortalidad materna.

\section{TECNOLOGÍAS Y LOS LÍMITES DE LOS DERECHOS: LOS DIU (CHILENOS) Y LA PÍLDORA ANTICONCEPTIVA}

En algunas de las primeras incursiones con la tecnología de planificación familiar, la curiosidad científica motivó la investigación y la experimentación con los cuerpos de las mujeres. Jaime Zipper, por ejemplo, uno de los médicos chilenos que trabajaba en hospitales públicos, constató, de primera mano que las mujeres intentaron limitar los embarazos. Por propia iniciativa, abrió la primera clínica de anticonceptivos en el Hospital Barros Luco de Santiago con tecnologías médicas de su propio diseño. En 1959, Zipper supo acerca de los problemas médicos que habían enfrentado los médicos con los Dispositivos Intra-Uterinos (DIU) probados por Gräfenberg. Inició una serie de experimentos y encontró un anillo, cuyo extremo suelto podía ser usado como una cola para la remoción. Con su invención en mano, puso el aviso "Clínica Anticonceptiva" en la puerta de su oficina, e inició una nueva etapa de investigación con el "anillo Zipper". Entre octubre de 1959 y junio de 1963, 3 mil mujeres llegaron a su oficina en el Hospital Barros Luco, donde fueron provistas del anillo. ${ }^{17}$

Las mujeres que vivían en la comunidad cercana al hospital fueron las primeras en probarlo, experimento que se apoyó estratégicamente en las relaciones tradicionalmente jerárquicas entre mujeres y médicos, entre profesional y paciente, así como entre hombres y mujeres. Aquéllas que dependían de los servicios públicos de salud raramen- te cuestionaban la autoridad médica. Si bien los tempranos esfuerzos de Zipper ayudaron a que los dispositivos de la primera planificación familiar estuviesen disponibles, reforzaron relaciones jerárquicas sociales y de género: médico y paciente, marido y esposa, hombre y mujer.

En los años sesenta también hubo otras tecnologías anticonceptivas, especialmente la píldora anticonceptiva -que transforma el debate sobre la regulación de la reproducción humana en el mundo entero. La disponibilidad de nueva tecnología más efectiva en el control de la natalidad tuvo implicancias revolucionarias. En 1962, se inició le venta de la píldora en Chile, con receta médica y solo a mujeres casadas. En 1968, las mujeres chilenas, casadas o solteras, pudieron, por primera vez, conseguir la píldora anticonceptiva en una farmacia local. Pero el acceso desigual a estas tecnologías, la limitada información disponible para algunas mujeres, y la dimensión política de la regulación de la fecundidad en la Guerra Fría, revelan que no podemos equiparar las tecnologías reproductivas con los derechos de las mujeres ni podemos ver las historias de la planificación familiar y los derechos reproductivos como historias de tecnología, o como las historias que permanecieron al margen de más amplias discusiones globales sobre los significados de la población y la regulación de la fertilidad.

Después de la Segunda Guerra Mundial, los temores del crecimiento explosivode la población, así como las tensiones de la Guerra Fría, añadieron nuevas dinámicas a la planificación familiar. Éstos tuvieron múltiples resultados: por una parte, proporcionaron un nuevo discurso que ayudó a justificar los programas de planificación familiar en el nombre del control de la población; por otra, las elecciones autónomas de las mujeres surgieron en un nuevo contexto cuando los planificadores de la población vincularon la planificación familiar al control de la población. Vemos que la tecnología anticonceptiva proporcionó una base importante para la maternidad voluntaria -pero la existencia de la píldora no garantizó automáticamente un aumento en el poder de las mujeres para decidir sobre el 
número y el intervalo entre uno y otro hijo, y no garantizó derechos reproductivos-. De hecho, el concepto de estos derechos aún estaba solamente "en construcción".

\section{DESARROLLOS DE LA PLANIFICACIÓN FAMILIAR EN CHILE: DE RESPONSABILIDADES Y DERECHOS}

El ginecólogo Gildo Zambra, quien trabajaba en la Asociación Chilena de Protección de la Familia (APROFA) en los años de 1970, me explicó en una entrevista que " $U n$ par de pioneros de la salud pública chilena, como Benjamín Viel y Hernán Romero, ya estaban empezando a hablar del tema [de derechos], porque leian mucho, se conectaban con el extranjero; ellos empezaron a introducir un poco la idea de que la mujer tenía derecho a regular su fecundidad". Explicó que él y sus colegas ginecólogos fueros testigos de las consecuencias de los abortos mal hechos, y ésta fue la razón por la cual empezaron a hablar de métodos anticonceptivos. Añadió: “...y no tanto por la necesidad de la mujer de realizarse como esposa, como madre, como trabajadora,... eso no nos llegaba en esa época, yo lo recuerdo muy bien". ${ }^{17}$

Los comentarios del Dr. Galán nos hacen recordar que el concepto de "derechos reproductivos" es relativamente nuevo a nivel mundial, y también en Chile. En 1968, durante la Conferencia Internacional de Derechos Humanos en Teherán, Irán, la planificación familiar fue definida por primera vez en un documento internacional, como el derecho humano fundamental de los padres para decidir sobre el número de hijos y los intervalos entre sus nacimientos. ${ }^{18}$ Encontramos las raíces de un nuevo discurso de derechos en la década de 1970, estimulado a través de un nuevo paradigma global de los derechos de las mujeres como derechos humanos. Activistas, políticos y académicos han denominado el año 1975 como un "motor para el cambio", considerándolo un hito en la historia de los derechos de las mujeres, dándole el crédito a las Naciones Unidas de declararlo el Año Internacional de la Mujer (AIM). En 1975, más de cinco mil personas de todo el mundo se juntaron en Ciudad de México para participar en la primera Conferencia Internacional de la Mujer de las Naciones Unidas o en la Tribuna de las Organizaciones No Gubernamentales (ONG) que convocó simultáneamente. Las participantes en la conferencia redactaron el borrador del Plan Mundial de Acción que definió la agenda para eliminar la discriminación en contra de las mujeres. También se extendió el Año Internacional de la Mujer a una Década de la Mujer (1975-85). ${ }^{19}$

Los delegados en conferencias internacionales produjeron otros documentos clave entre la década de los sesenta y los años noventa. En 1979, la Convención sobre la eliminación de todas las formas de discriminación contra la mujer (CEDAW) de 1979, ayudó a presionar a los gobiernos para que se comprometieran a elaborar códigos que sancionaran la equidad de género. Los líderes nacionales interesados podían elegir ratificar la propuesta de CEDAW o firmar una versión modificada. En 1993, la Declaración y el Programa de Acción de la Conferencia Mundial de los Derechos Humanos celebrada en Viena reconocieron oficialmente que "los derechos de las mujeres son derechos humanos”. En 1994, la Conferencia Mundial de las Naciones Unidas sobre Población y Desarrollo en El Cairo definió y reconoció los "derechos reproductivos" tanto de hombres como de mujeres, especificando que: "la salud reproductiva... supone que las personas son capaces de tener una vida sexual satisfactoria y que tienen la capacidad de reproducirse y la libertad de decidir si es así, cuándo y con qué frecuencia hacerlo". Para la cuarta Conferencia Mundial de Mujeres de las Naciones Unidas llevada a cabo en Beijing en 1995, los derechos de las mujeres se habían convertido en una parte central del lenguaje y la agenda oficial de los derechos humanos internacionales. Los nuevos paradigmas globales de derechos no tienen consecuencias jurídicas directas en el nivel del Estado-nación, pero proponen normas clave, modelos que un gobierno puede aprobar, parcialmente ratificar, o ignorar por completo. Y estas normas, incluso si no tienen ningún poder directo para cambiar las leyes de los Estados, representan pautas importantes que permiten que 
los ciudadanos puedan solicitar cambios en la política del Estado-nación.

Galán, y otros médicos antes que él, corroboró el peso que el problema del aborto tuvo para esforzarse en ofrecer nuevos métodos de planificación familiar a las mujeres. El obstetra Dr. Aníbal Faúndes, por ejemplo, fue un joven interno en un hospital público de Santiago, que asistió a las mujeres que buscaron atención de emergencia debido a abortos inducidos. Más de 50 años después, aún recuerda su angustia en el año 1953: "El hecho de presenciar el dolor físico y psicológico de mujeres jóvenes y mayores, escuchar sus historias, verlas sufrir durante semanas y observarlas morir o sobrevivir gravemente mutiladas, significó una fuerte motivación para consagrar una gran parte de mi vida profesional a encontrar un modo de mitigar sus padecimientos". ${ }^{20} \mathrm{La}$ epidemia del aborto provocado, bien documentada en la época de los años sesenta, reveló un conjunto de preocupaciones reales de los médicos, quienes buscaron prevenir la mortalidad materna al convertir la maternidad en una opción, dando el poder de decisión a la mujer.

Sin embargo, controlar la "sobrepoblación" fue la otra razón por la cual los anticonceptivos se hicieron disponibles para la mujer. En 1966, el Dr. Viel publicó su propio estudio, de amplia recepción, sobre la experiencia de la "explosión demográfica" en América Latina. ${ }^{21}$ Documentando el descenso de la mortalidad que comenzó en Santiago alrededor de 1937, Viel resaltó el inicio de una explosión demográfica de proporciones peligrosas. El avance de la práctica médica y las nuevas tecnologías ayudarían a una mayor supervivencia de la población, e irónicamente, requerirían de una nueva atención dado el tamaño de la población. Viel vinculaba el desafío del subdesarrollo a la sobrepoblación, sugiriendo que el excesivo crecimiento demográfico no solamente competía por los limitados recursos, sino que también estancaba el progreso económico.

Tomó una posición neomalthusiana, y, simultáneamente, pudo asegurar un apoyo internacional para sus proyectos en Chile. Benjamín Viel demostró sus capacidades de salubrista y hombre político; él sabía vencer los obstáculos y dificultades de la legitimación social de la planificación familiar en los años de 1960. Aunque no podía convencer a todos de que "Malthus tenía razón", su enfoque aseguró el apoyo de la Fundación Rockefeller y la IPPF (International Planned Parenthood Federation/ Federación Internacional de Paternidad Planificada). En las actividades de Benjamín Viel también encontramos un mundo de colaboración entre doctores y científicos atravesando barreras económicas y políticas. En 1967, Viel y otros médicos habían presionado exitosamente para ser los anfitriones de la Octava Conferencia Internacional de la Federación Internacional de Planificación Familiar, reunión que por primera vez tenía lugar en una nación latinoamericana -bajo el principio de "Paternidad conscientedeber y derecho humano"-. El camino de la historia del desarrollo de la planificación familiar como "deber" de la mujer está más presente que su transformación desde un supuesto "deber" a un "derecho". 22

Extraordinariamente, los demócrata cristianos bajo la presidencia de Eduardo Frei Montalva (1964-1970) no solo apoyaron programas de planificación familiar, además contribuyeron activamente a la relación laboral entre el Servicio Nacional de Salud (SNS) y las iniciativas privadas de planificación familiar instigadas por Viel y la Asociación Chilena de Protección de la Familia, APROFA. Programas de planificación familiar y las campañas de información de APROFA, que promovían la planificación familiar como una forma de proteger el bienestar de la familia, encajaban bien en la plataforma política de la Democracia Cristiana. Empleados en el sistema de salud pública alentaron a las mujeres a utilizar los servicios de APROFA así como los del Servicio Nacional de Salud (SNS), y trabajaban en colaboración con esta asociación. Encontramos la prueba de los resultados exitosos de esta colaboración en cifras: en 1966, 102 centros de planificación familiar se enfocaron en las necesidades de las mujeres en todo el país, y más de 58 mil mujeres recibieron anticonceptivos gratuitamente. En 1967, en el tercer año de gobierno de la Democracia 
Cristiana, éste aumentó los fondos destinados a los servicios de planificación familiar para cubrir adicionalmente a 100 mil mujeres. ${ }^{23}$

\section{LO PERSONAL ES POLÍTICO: CONSIDERACIONES DE LA GUERRA FRÍA}

La Guerra Fría provocó nuevas tensiones entre múltiples representaciones de las derechas e izquierdas políticas.

En este contexto, volvemos -de nuevoal viejo conflicto entre Karl Marx y Thomas Malthus. En el discurso político de los portavoces de la izquierda latinoamericana, las posiciones neomalthusianas fueron fácilmente equiparadas con posiciones anticomunistas y con el deseo de controlar el tamaño de las poblaciones supuestamente rebeldes. Los defensores del control de la población, procedentes frecuentemente de los Estados Unidos, proporcionaron amplias evidencias para que los izquierdistas llegaran a estas conclusiones. Sin embargo, ni las voces de la izquierda ni las de la derecha se preocupaban por el interés de las mujeres individuales o de las parejas que buscaron planificar sus embarazos y familias. La tensión de la Guerra Fría -entre la izquierda y la derecha- limitó los espacios para la práctica de los derechos reproductivos.

\section{EL COMPONENTE ANTICOMUNISTA DE LAS POLÍTICAS DE POBLACIÓN}

Después de la Segunda Guerra Mundial, los encargados (o autonominados) norteamericanos en políticas de población dejaron claro que el poder reproductivo de las mujeres era profundamente político -ligado también a una supuesta amenaza de revolución. El general norteamericano William H. Draper, por ejemplo, miembro fundador del Population Crisis Committee, (Comité de Crisis en Población) divulgó temores sobre revoluciones que se extendían y de comunistas tomando el poder. En anuncios y campañas informativas, su comité, y otros ciudadanos preocupados también, presentaron "naciones hambrientas" llenas de gente que podría encenderla "bomba poblacional". Los pobres podrían "poner en peligro" o "amenazar la paz del mundo" cuando el descontento creciente hiciera erupción en una revolución social. ${ }^{24}$ Los anuncios advertían que "la marea cada vez mayor de la humanidad exige que la controlemos, para no ser sumergidos en ella junto con nuestros valores de civilización". ${ }^{25}$

Acelerado por la realidad de la Revolución cubana, la supuesta amenaza de la agitación revolucionaria parecía tan real como la falta en el suministro de alimentos debido a un crecimiento descontrolado de la población. En una carta abierta dirigida al presidente Lyndon Johnson, patrocinada por el fondo Hugh Moore, ciudadanos norteamericanos ansiosos advirtieron que mucho estaba en riesgo: "La explosión demográfica inevitablemente llevará al caos y a la lucha [en los Estados Unidos] [...]y en el extranjero". Recomendaron tomar medidas para evitar otros Cuba y Vietnam y desarrollos que podrían destruir los valores civilizados en el molino comunista. ${ }^{26}$ En 1965 , la declaración pública del presidente Johnson sobre el control de la población ilustraba el vínculo neomalthusiano -ahora entre el subdesarrollo y la sobrepoblación-: "Menos de cinco dólares invertidos en el control de la población equivalen a cien dólares invertidos en el desarrollo económico". ${ }^{27}$

Ciertamente, el discurso de los científicos y políticos en los Estados Unidos enfatizaba justificaciones neomalthusianas a un nivel tan alto que frecuentemente distraían del potencial libertador que los programas de planificación familiar podrían proporcionar a las mujeres de manera individual. Algunos científicos norteamericanos enfatizaban que la supuesta sobrepoblación del llamado "Tercer Mundo" era uno de los incentivos principales para invertir en la indagación de un anticonceptivo hormonal efectivo como la píldora. El biólogo Gregory Pincus, por ejemplo, afirmaba que la investigación acerca de los primeros experimentos con la píldora tenía como finalidadayudar a abordar el problema de la sobrepoblación, especialmente en las naciones en desarrollo. En su propio recuento de los eventos que llevaron a la investigación científica de la píldora anticonceptiva, habla inclusive de la visita de la enfermera y activista a favor de la planificación familiar 
Margaret Sanger -quien lo alentó, con un presentimiento de crisis, a que ayudara a desarrollar un método a toda prueba para controlar el crecimiento "fuera de control" de la humanidad-. ${ }^{28}$ Ciertamente, para algunos, los resultados de las investigaciones científicas parecían más urgentes que nunca en el contexto de las tensiones políticas causadas por el descontento del rápido incremento de las masas empobrecidas.

Aún más, en la década de los años sesenta, John D. Rockefeller tercero, fundador del Population Council (Consejo para la Población) y muy dedicado al desarrollo de los programas de salud y población de la Fundación, viajó a múltiples países latinoamericanos para buscar aliados que apoyaran su esfuerzo por resolver el "problema de la población”. Pedía a los jefes de gobierno que apoyaran su "Declaración sobre Población de los Jefes de Gobierno", la cual presentó, en primer lugar, al secretario general de las Naciones Unidas en 1966. Aquéllos que apoyaron la declaración se suscribían a la idea de que "el problema de la población debe ser reconocido como elemento principal en una planificación nacional de largo alcance si los gobiernos quieren lograr sus objetivos económicos y cumplir las expectativas de su gente [...] que la oportunidad de decidir el número y el lugar de los hijos es un derecho humano básico; [y] que la paz duradera y significativa depende en gran medida de la manera en la que los retos para el crecimiento de la población sean alcanzados". ${ }^{29}$

A pesar de que Rockefeller reconoció que la planificación familiar era un derecho humano básico, continuó poniendo énfasis en los peligros de la sobrepoblación y ayudó a promover políticas que requerían la vigilancia sobre la fertilidad de las mujeres. Alentó el establecimiento de políticas de control de la población, especialmente en regiones con un rápido crecimiento de ésta. En este contexto, los beneficios de los programas de planificación que comenzaron a funcionar en muchos países latinoamericanos a mediados de los años sesenta, siguieron frecuentemente afectados por disputas ideológicas y rivalidades entre políticas de derecha y de izquierda.

\section{POSICIONAMIENTOS IZQUIERDISTAS: EL RECHAZO (DOGMÁTICO) DEL NEOMALTHUSIANISMO}

Algunos intelectuales marxistas y militantes de la izquierda no solo condenaron los proyectos neomalthusianos como una nueva amenaza imperialista que significaba aplastar a la izquierda latinoamericana $-y$ dieron un nuevo nombre a las iniciativas de planificación familiar: control de la población-. En 1971, el intelectual uruguayo Eduardo Galeano se refirió al tema de la planificación familiar en la introducción a su libro Las venas abiertas de América Latina:

Los Estados Unidos [...] se preocupan como nadie por difundir e imponer [...] la planificación familiar. No solo el gobierno; también Rockefeller y la Fundación Ford padecen pesadillas con millones de niños que avanzan, como langostas, desde los horizontes del Tercer Mundo. Platón y Aristóteles se habían ocupado del tema antes que Malthus y McNamara; sin embargo, en nuestros tiempos, toda esta ofensiva universal cumple una función bien definida: se propone justificar la muy desigual distribución de la renta entre los países y entre las clases sociales, convencer a los pobres de que la pobreza es el resultado de los hijos que no se evitan y poner un dique al avance de la furia de las masas en movimiento y rebelión. Los dispositivos intrauterinos compiten con las bombas y la metralla, en el sudeste asiático, en el esfuerzo por detener el crecimiento de la población de Vietnam. En América Latina resulta más higiénico y eficaz matar a los guerrilleros en los úteros que en las sierras o en las calles. [...] Muerta y enterrada la Alianza para el Progreso, el Imperio propone ahora, con más pánico que generosidad, resolver los problemas de América Latina eliminando de antemano a los latinoamericanos. ${ }^{30}$

Galeano, con la fuerza de su voz como uno de los escritores más conocidos de la izquierda política latinoamericana, presenta la proliferación 
de los programas de planificación familiar en la región como nada más (o menos) que una ofensiva global controlada por los Estados Unidos. En esta percepción, el ánimo de los programas de planificación familiar cumple una función bien definida.

El ataque de Galeano a la planificación familiar reflejaba un consenso amplio entre sectores de la izquierda latinoamericana. José Consuegra, intelectual y economista marxista colombiano, condenaba a los neomalthusianos en su libro El control de la natalidad como arma del imperialismo. Ligando el control de la población a los intereses de clase de los burgueses, enfatizando el componente antirrevolucionario del pensamiento neomalthusiano,31 afirma que la noción de la sobrepoblación se convierte en una excusa para la pobreza de las clases trabajadoras, y el control de la natalidad solo sirve para prolongar artificialmente un sistema económico que produce subdesarrollo y reproduce jerarquías de clase.

Algunos sectores de la izquierda chilena recogieron este discurso crítico, y desarrollaron uno propio. Consideraron el asunto de la planificación familiar en la campaña electoral de 1970, y acusaron a los planificadores familiares de venderse a los imperios extranjeros para controlar y limitar poblaciones. En agosto de 1970, el periódico comunista El Siglo fue tan lejos como acusar a los funcionarios de salud de Chile en general y al pionero de la planificación familiar, Dr. Benjamín Viel, en particular, de "encabezar una campaña de origen norteamericano para presionar a la clase trabajadora y a las mujeres campesinas para limitar los nacimientos". El Siglo describió cómo Viel, supuestamente, abusó de la vulnerabilidad de los chilenos pobres e implementó medidas de control de la población. Además, el periódico lo acusó de manipular a médicos y parteras para promover la planificación familiar mediante el pago con dinero sucio del exterior. Después de todo, afirmaba El Siglo, la Fundación Rockefeller y los norteamericanos que controlaban la población tenían intereses políticos y económicos para limitar el tamaño de la población en Chile. Otro artículo de El Siglo se dirigía al “Doctor 'Malthus' Viel” en el encabezado de un artículo, y llamaba a las iniciativas de planificación familiar armas económicas y políticas usadas en contra de los chilenos. ${ }^{32}$ En esta lógica, ni las izquierdas políticas ni los defensores de posiciones neomalthusianas, crearon un espacio para las consideraciones respecto a las necesidades de las mujeres, y para una defensa a su derecho de tomar decisiones sobre embarazo y maternidad.

\section{EPÍLOGO}

Las realidades de la historia de la planificación familiar en Chile indican que el acceso a anticonceptivos fue un primer paso hacia la defensa de los derechos reproductivos de las mujeres chilenas. No obstante, continuaba siendo una negociación no resuelta entre control y derecho.

En la época de los años sesenta, los dispositivos intrauterinos, los DIU, se convirtieron en el método anticonceptivo más extendido en Chile, popular entre los médicos porque era efectivo en costo y relativamente fiable después de su inserción. Entre 1964 y 1969,los índices de nacimientos disminuyeron en un 33 por ciento. El número de mujeres hospitalizadas por aborto había disminuido por al menos la misma tasa en el mismo período y área geográfica. Viel enfatizó que estas bajas eran significativamente más grandes que aquellas documentadas "en países en donde los programas de planificación familiar han estado usando 'píldoras' como el mayor anticonceptivo”. Viel concluía que el éxito se debía a la efectividad del uso del DIU. Él también sabía que los índices de continuidad estaban directamente relacionados con el control de los doctores en la inserción del DIU, un control que no tenían con el uso de la píldora. ${ }^{33}$

Los contextos de las negociaciones sobre quiénes controlaban las vidas reproductivas de las mujeres en la década de los sesenta y en décadas posteriores también justifican mi perspectiva específica: En este estudio de la historia y la política de los derechos reproductivos, me refiero a la historia de los derechos de las mujeres, porque la evidencia histórica demuestra que sus derechos reproductivos han sido violados con mayor frecuencia, $y$, 
seguramente, con más violencia que los derechos reproductivos de los hombres. Encontramos evidencia de estas violaciones en la historia global, y también en Chile, y podemos demostrar que se justifica este enfoque. Los acontecimientos históricos bajo la dictadura chilena, por ejemplo, no solo proporcionan una amplia evidencia para demostrar que tenemos que prestar atención a las violaciones de los derechos reproductivos de las mujeres, pero también confirman que la idea de progreso lineal, y de la expansión progresiva de los derechos, es engañosa en el mejor de los casos.

La experiencia de una mujer demuestra, nuevamente, que la regulación de la fertilidad y las restricciones de los derechos reproductivos están mediadas (frecuentemente controladas) por las metas políticas del 'liderazgo de la Nación'. En 1981, la señora Leontina Huetel debe de haberse asustado por la noticia recibida en el consultorio de su doctor en Santiago. Éste le dijo: "Lo que le está pasando, señora, es que usted está embarazada". Estaba utilizando el Dispositivo Intrauterino (DIU) y recientemente se había asegurado de su correcta colocación. Solo que ahora, cuando ya era tarde, había descubierto que lo que el doctor le había hecho realmente era retirarle el anticonceptivo sin su consentimiento. El retiro de los DIU en contra de la voluntad de las mujeres se convirtió en una poderosa "leyenda urbana" (con un trasfondo verdadero) bajo la dictadura. Si bien el número de retiros sin autorización no puede ser documentado, las mujeres comenzaron a evitar las visitas a los doctores como resultado de eso. Muchas cancelaron citas a los centros de salud locales por miedo al "cuidado" que podrían recibir.(17, p.142)

Con la dictadura, los cambios políticos específicos contribuyeron a un ambiente en el que la maternidad como opción se convirtió en una tarea cada vez más difícil. En 1973, el Ejército comenzó a descentralizar el sistema de salud pública y puso a los consultorios locales bajo el control municipal. Después, entre 1979 y 1985, los militares implementaron temporalmente una política pro-nacimientos, impidiendo a los médicos que proporcionaran servicios de planificación familiar. Muchos de ellos ya no pusieron los anticon- ceptivos a disposición de las mujeres. Finalmente, en 1989, el gobierno militar eliminó el derecho legal de las mujeres al aborto terapéutico; esta criminalización del aborto sigue teniendo consecuencias fatales, afectando, con más fuerza, a las mujeres jóvenes y a las mujeres sin recursos. En resumen, el régimen desarrolló un conjunto de leyes interrelacionadas para controlar la vida reproductiva de las mujeres chilenas, ahora subyugadas a los intereses geopolíticos de la dictadura.

Durante los primeros años de la redemocratización, la defensa tanto de los derechos reproductivos en general como de los derechos de las mujeres en particular, resultó ser una tarea difícil debido a dos cambios finales, y profundamente contradictorios, implementados por la dictadura: la prohibición de aborto terapéutico y la firma de la Convención sobre la Eliminación de todas las Formas de Discriminación contra la Mujer (Convention on the Elimination of All Forms of Discrimination against Women, CEDAW).La nueva ilegalidad de los abortos terapéuticos de hecho violaba los mismos principios definidos por CEDAW, pero solo el primero y no el segundo tuvo consecuencias de obligación legal en Chile.

En los años transcurridos desde la redemocratización, ha quedado claro que las mujeres pobres, así como las adolescentes, son más propensas a sufrir las consecuencias del sistema de salud en declive, de la educación sexual inadecuada y la falta de acceso al aborto legal y terapéutico. En la década de 1960, funcionarios de salud chilenos proporcionaron estudios pioneros acerca de una epidemia de aborto inducido, y señalaron el camino en las campañas de salud al abrir clínicas de planificación familiar a lo largo de todo el país. En la década de 1990 y en el nuevo milenio, las mujeres chilenas han tenido que enfrentarse a las consecuencias a largo plazo de la política militar y han tenido que encontrar formas de sobrevivir a los retos de salud que recuerdan a los de principios del siglo XX. Hoy, la mortalidad materna y las complicaciones como resultado de abortos ilegales representan una de las crisis de salud pública más alarmantes en la nación chilena. 


\section{REFERENCIAS}

1. Harvey D. Population, Resources, and the Ideology of Science. Econ Geog. 1974; 50(3): 256-277

2. Mattelart A. Prefiguración de la ideología burguesa. Lectura ideológica de una obra de Malthus," El Trimestre Económico. 1971; 38 (149-1): 145-188.

3. Malthus $\mathrm{T}$ [1798]. An Essay on Population. (New York, Oxford University Press, 2008), 61.

4. Foster JB. Malthus's essay on population at age 200. En: Bellamy Foster J. Ecology against Capitalism. New York: Monthly Review Press; 2002. p. 137.

5. Simon JL. The Economics of Population Classic Writings [en línea] New Brunswick, N.J., U.S.A.: Transaction Publishers, 2004 [consultado en 2013]. Disponible en: http:// search.epnet.com/direct.asp?db=buh\&jid= $\% 22 \mathrm{PWV} \% 22 \&$ scope $=$ site.

6. Wiltgen R. Marx and Engels on Malthus and Population: a Reconstruction and Reconsideration. Quar Rev Econ Buss [en línea] 1981; 21: 4: 107-26 [consultado 2012]. Disponible en: http://growthconf.ec.unipi.it/ papers/Brezis.pdf

7. Armus D. Disease in the Historiography of Modern Latin America. En: Armus, D, ed. Disease in the history of Modern Latin America: from Malaria to AIDS. Durham: Duke University Press; 2003. p. 1-24.

8. Gaete J. La historia y los grandes médicos del pasado en Chile: Sentido de una indagación. Cuad Médico Soc. 2010; 50(3): 208-219.

9. Connelly M. Fatal Misconception: the struggle to control world population. Cambridge, Mass: Belknap Press of Harvard University Press; 2008.

10. Shephard B. Running the obstacle course to sexual and reproductive health: lessons from Latin America. Westport CT: Praeger; 2006.

11. Del Campo A. La nación en peligro: el debate médico sobre el aborto en Chile en la década de 1930. En: Zárate, María Soledad; ed. Por la salud del cuerpo, historia y políticas sanitarias en Chile. Santiago: Alberto Hurtado; 2008. p. 133-188.

12. Romero H, Medina E, Vildósola J. Aportes al conocimiento de la procreación. Rev Chil Hig Med Prev. 1953; 15(3-4): 73-90.
13. Armijo R, Monreal T. Epidemiology of Provoked Abortion in Santiago, Chile. J Sex Res. 1965; 1(152): 143-159.

14. Requena M. Condiciones determinantes del aborto inducido. Rev Méd Chile. 1966; 94: 714-722.

15. Proceedings of the Eighth International Conference of the International Planned $\mathrm{Pa}$ renthood Federation, Santiago, Chile, 9-15 April 1967, London, IPPF, 1967; p. 143.

16. Plaza S, Briones $\mathrm{H}$. El aborto como problema asistencial. Rev Méd Chile. 1963; 91(4): 294-97.

17. Pieper Mooney J. The Politics of Motherhood: Maternity and Women's Rights in Twentieth-Century Chile. Pittsburgh, Pa: University of Pittsburgh Press; 2009).

18. Dr. Gildo Zambra, comunicación personal. Naciones Unidas. Proclamación de Teherán. Conferencia Internacional de Derechos Humanos en Teherán. Párrafo 16; 13 de Mayo de 1968.

19. United Nations. World Plan of Action for the Implementation of the Objectives of the International Women's Year: a summarized version. New York: United Nations, Centre for Economic and Social Information; 1976.

20. Faúndes A, Barzelatto J. El drama del aborto: en busca de un consenso. Santiago: LOM Ediciones; 2007.

21. Viel B. La explosión demográfica. ¿Cuántos son demasiados? Santiago: Ediciones de la Universidad de Chile; 1966.

22. Federación Internacional de Planificación de la Familia. Actas de la Octava Conferencia Internacional de la Federación Internacional de Planificación de la Familia, Santiago de Chile, 9-15 de abril de 1967: Paternidad consciente-deber y derecho humanos. Hertfordshire, Inglaterra: Stephen Austin and Sons; 1968.

23. International Planned Parenthood Federation and Hankinson. Proceedings of the Eighth International Conference of the International Planned Parenthood Federation; 9-15 April, 1967; Santiago, Chile.

24. New York Times. 23 de febrero de 1969, sec. IV, 5, y 9 de febrero de 1969, sec. IV, 5.

25. New York Times, 9 de febrero de 1969, sec. IV, 5, y 23 de febrero de 1969, sec. IV, 5; New York Times, 14 de diciembre de 1969, 56. 
26. New York Times, 13 de diciembre de 1964, sec. IV, 5.

27. Lyndon Johnson. Discurso presidencial ante las Naciones Unidas. 25 de junio de 1965.

28. Pincus G. The Control of Fertility. New York: Academic Press; 1965.

29. Statement on Population from World Leaders [en línea] [consultado en 2012]. Disponible en: http://www.popcouncil.org/ mediacenter/popstatement.html

30. Galeano, E., Las venas abiertas de América Latina (Madrid: Siglo XXI de España Editores, 2003), 9.
31. Consuegra, J., El control de la natalidad como arma del imperialismo. Buenos Aires: Editorial Galerna, 1969.

32. El Siglo, 4 y 15 de agosto de 1970.

33. Viel B. Resultados de un Programa de Anticonceptivos Basado en DIUs en Chile en Proceedings of the Family Planning Research Conference; a multidisciplinary approach, Exeter, England, September 27-28, 1971. Edited by Alfredo Goldsmith [and] Robert Snowden. 